\title{
Numerical simulation of high-resolution azimuthal resistivity laterolog response in fractured reservoirs
}

\author{
Shao-Gui Deng ${ }^{1} \cdot \mathrm{Li} \mathrm{Li}^{1} \cdot$ Zhi-Qiang $\mathrm{Li}^{2} \cdot \mathrm{Xu}$-Quan $\mathrm{He}^{3} \cdot \mathrm{Yi}-\mathrm{Ren} \mathrm{Fan}^{1}$
}

Received: 8 July 2014/Published online: 9 April 2015

(c) The Author(s) 2015. This article is published with open access at Springerlink.com

\begin{abstract}
The high-resolution azimuthal resistivity laterolog response in a fractured formation was numerically simulated using a three-dimensional finite element method. Simulation results show that the azimuthal resistivity is determined by fracture dipping as well as dipping direction, while the amplitude differences between deep and shallow laterolog resistivities are mainly controlled by the former. A linear relationship exists between the corrected apparent conductivities and fracture aperture. With the same fracture aperture, the deep and shallow laterolog resistivities present small values with negative separations for low-angle fractures, while azimuthal resistivities have large variations with positive separations for high-angle fractures that intersect the borehole. For dipping fractures, the variation of the azimuthal resistivity becomes larger when the fracture aperture increases. In addition, for high-angle fractures far from the borehole, a negative separation between the deep and shallow resistivities exists when fracture aperture is large as well as high resistivity contrast exists between bedrock and fracture fluid. The decreasing amplitude of dual laterolog resistivity can indicate the
\end{abstract}

Shao-Gui Deng

dengshg@upc.edu.cn

1 School of Geosciences, China University of Petroleum, Qingdao 266580, Shandong, China

2 China Research Institute of Radiowave Propagation, Xinxiang 453003, Henan, China

3 China Petroleum Southwest Oil and Gas Field Branch, Chengdu 610051, Sichuan, China

Edited by Jie Hao aperture of low-angle fractures, and the variation of the deep azimuthal resistivity can give information of the aperture of high-angle fractures and their position relative to the borehole.

Keywords High-resolution azimuthal resistivity laterolog · Fractured reservoir - Fracture dipping angle . Fracture aperture $\cdot$ Fracture dipping direction

\section{Introduction}

Fracture is the smallest and the most complex structure in the crust. It can not only increase the pore space and permeability, but also control the formation, distribution, and capacity of oil and gas in place (Jiang et al. 2004; Zeng et al. 2007; Zhang et al. 2009; Weng et al. 2011; Nie et al. 2012; Kuchuk and Biryukov 2014; Reynolds et al. 2014; Yao et al. 2013; Zhao et al. 2014). Fracture identification and quantitative characterization are keys to effective exploration of fractured reservoirs (Bourbiaux 2010; Sun et al. 2011). Dual laterolog is widely used to study fracture development and fracture porosity because of good current focusing and detectability of fractured formation (Sibbit and Faivre 1985; Li et al. 1996; Deng and Li 2009; Noroozi et al. 2010; Le et al. 2011; Ja'fari et al. 2012; Deng et al. 2013). However, dual laterolog cannot accurately reflect complex heterogeneity and anisotropy in fractured reservoirs. Formation MicroScanner Image (FMI) figures can provide visual displays of sidewall geological characteristics of fractures, caves, etc. But shallow investigation depth limits its further application (Shen et al. 2009; Dershowitz et al. 2010; Sausse et al. 2010; Yang et al. 2011; Deng et al. 2012; Moinfar et al. 2010; Yun et al. 2013). Azimuthal resistivity imager (ARI) and high-resolution azimuthal 
laterolog sonde (HALS) were earlier proposed to study three-dimensional distribution of resistivity surrounding the borehole. However, the logging response mechanism is not well understood and application examples about fractures are rare (Faivre 1993; Davies et al. 1994; Smits et al. 1995; Yang and Tao 1999; Karim et al. 2013; Olsen et al. 2014). This study aims to implement numerical simulation of the high-resolution azimuthal resistivity laterolog (HARL), in order to combine the radial detection of dual laterolog and azimuthal detection around the borehole, and then corresponding logging response characteristics and identification method of fractures are investigated to aid fractured reservoir evaluation.

\section{Three-dimensional finite element model of fractured reservoirs}

\subsection{Fundamental theory}

HARL can provide two measurement modes, highresolution dual laterolog mode and azimuthal resistivity

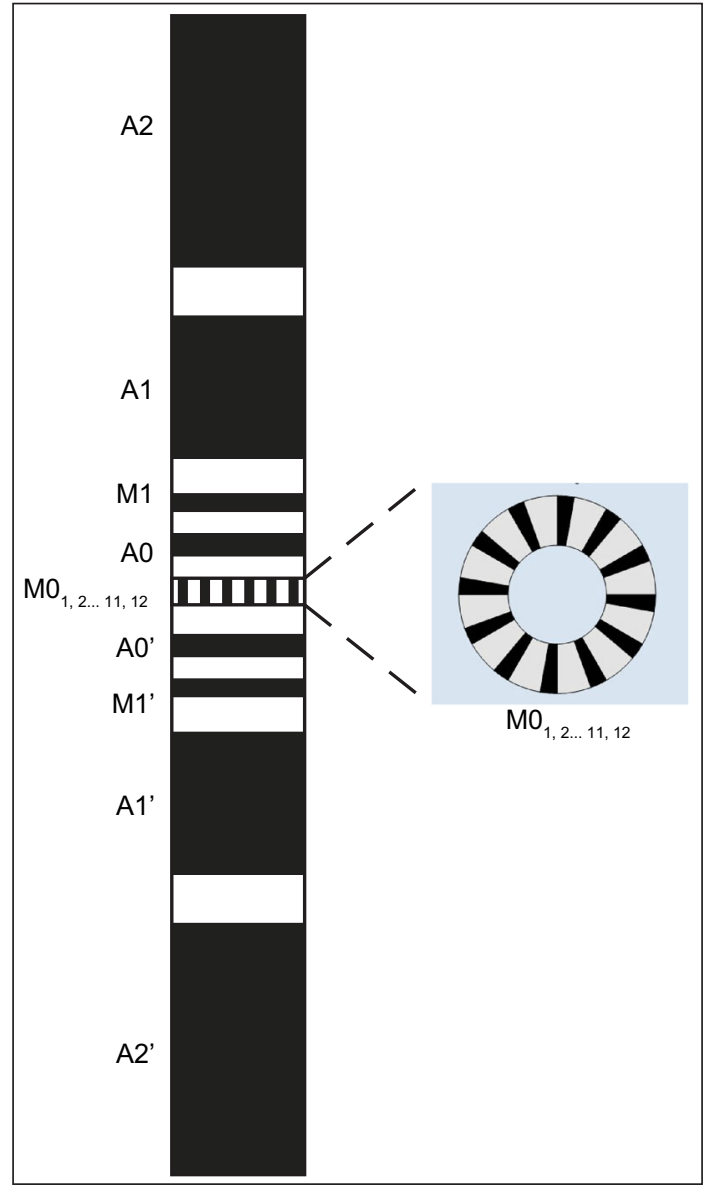

Fig. 1 High-resolution azimuthal resistivity laterolog measurement mode. As shown in Fig. 1, differing from the conventional dual laterolog, the main current electrode of high-resolution dual laterolog mode is divided into A0 and $\mathrm{A}^{\prime}$, and then an electrical potential guiding electrode (M0) is added between $\mathrm{A} 0$ and $\mathrm{A}^{\prime}$. According to the potential difference of three electrical potential guiding electrodes, the instrument constantly adjusts the focusing voltage. This configuration not only improves the vertical resolution, but also significantly reduces the instrument length. Azimuthal resistivity measurement mode is achieved by the electric potential difference between 12 azimuthal electrodes disposed in the electrical potential guiding ring (M0). The angle of a single azimuthal electrode is $10^{\circ}$. The angle between the center axes of two adjacent azimuthal electrodes is $30^{\circ}$. Therefore, the HARL can simultaneously measure high-resolution dual laterolog and azimuthal resistivity and then obtain two images of shallow and deep investigation depth, respectively.

High-resolution dual laterolog mode sets the electrical potential equal between the average value of 12 azimuthal electrodes and the two electrical potential guiding electrodes $\left(\mathrm{M} 1, \mathrm{M1}^{\prime}\right)$, then the expression formulas of high-resolution dual laterolog mode are obtained as follows:

HARLd $=K_{\mathrm{d}} \frac{U_{\mathrm{d}(\mathrm{M} 1)}}{I_{\mathrm{d}(\mathrm{A} 0)}+I_{\mathrm{d}\left(\mathrm{A} 0^{\prime}\right)}}$
HARLs $=K_{\mathrm{s}} \frac{U_{\mathrm{s}(\mathrm{M} 1)}}{I_{\mathrm{s}(\mathrm{A} 0)}+I_{\mathrm{s}\left(\mathrm{A} 0^{\prime}\right)}}$.

Based on the potential difference between the main current electrode and the 12 azimuthal electrodes, the expression formulas of azimuthal resistivity measurement mode can be obtained as follows:

$$
\begin{gathered}
\operatorname{HARLd}(i)=K_{\mathrm{d}} \frac{U_{\mathrm{d}(\mathrm{M} 1)}}{I_{\mathrm{d}(\mathrm{A} 0)}+I_{\mathrm{d}\left(\mathrm{A} 0^{\prime}\right)}} \frac{\sum_{i=1}^{12} \Delta U_{\mathrm{d}\left(\mathrm{M} 0_{i} \mathrm{~A} 0\right)}}{12 \Delta U_{\mathrm{d}\left(\mathrm{M} 0_{i} \mathrm{~A} 0\right)}} \\
i=1,2, \ldots, 12 \\
\operatorname{HARLs}(i)=K_{\mathrm{s}} \frac{U_{\mathrm{s}(\mathrm{M} 1)}}{I_{\mathrm{s}(\mathrm{A} 0)}+I_{\mathrm{s}\left(\mathrm{A} 0^{\prime}\right)}} \frac{\sum_{i=1}^{12} \Delta U_{\mathrm{s}\left(\mathrm{M}_{i} \mathrm{~A} 0\right)}}{12 \Delta U_{\mathrm{s}\left(\mathrm{M} 0_{i} \mathrm{~A} 0\right)}} \\
\quad i=1,2, \ldots, 12,
\end{gathered}
$$

where $K_{\mathrm{d}}$ and $K_{\mathrm{s}}$ are coefficients of instrument in deep and shallow laterologs; $U_{\mathrm{d}(\mathrm{M} 1)}$ and $U_{\mathrm{s}(\mathrm{M} 1)}$ are potential values of electrical potential guiding electrode (M1) in deep and shallow laterologs; $I_{\mathrm{d}(\mathrm{A} 0)}, I_{\mathrm{s}(\mathrm{A} 0)}, I_{\mathrm{d}\left(\mathrm{A}^{\prime}\right)}$, and $I_{\mathrm{s}\left(\mathrm{A}^{\prime}\right)}$ are current values of main current electrodes $\left(\mathrm{A} 0, \mathrm{~A} 0^{\prime}\right)$ in deep and shallow laterologs; $\Delta U_{\mathrm{d}\left(\mathrm{M}_{i} \mathrm{~A} 0\right)}$ and $\Delta U_{\mathrm{s}\left(\mathrm{M}_{i} \mathrm{~A} 0\right)}$ are potential difference between main current electrodes (A0, $\left.\mathrm{A} 0^{\prime}\right)$ and the azimuthal electrode $\left(\mathrm{MO}_{i}\right)$ in deep and shallow laterologs, respectively. 


\subsection{Calculation principle}

Assuming that a fractured reservoir consists of fractures and bedrock and that the fractures exist in the form of fracture groups, we use the plane model of parallel fractures (Zeng et al. 2007). As shown in Fig. 2, the conductivities of bedrock and fracture fluid are $\sigma_{\mathrm{b}}$ and $\sigma_{\mathrm{f}}$ and the aperture and dipping angle of each fracture are $h$ and $\Omega$, respectively. The response of HARL is a comprehensive effect of all media, including fractures and bedrock within the detection range. Assuming that the potential distribution generated by HARL around the borehole is $U$, the potential gradient of bedrock can be expressed as $\nabla U$, and the potential gradient in any fracture plane can be decomposed into a normal component $\left(\boldsymbol{E}_{\mathrm{bn}}\right)$ and a tangential component $\left(\boldsymbol{E}_{\mathrm{bt}}\right)$, which are given by

$$
\begin{aligned}
& \boldsymbol{E}_{\mathrm{bn}}=\boldsymbol{e}_{\mathrm{n}}\left(-\nabla U \cdot \boldsymbol{e}_{\mathrm{n}}\right) \\
& \boldsymbol{E}_{\mathrm{bt}}=\left(-\nabla U \cdot \boldsymbol{e}_{\mathrm{t}}+\boldsymbol{e}_{\mathrm{n}}\left(\nabla U \cdot \boldsymbol{e}_{\mathrm{n}}\right) \cdot \boldsymbol{e}_{\mathrm{t}}\right) \cdot \boldsymbol{e}_{\mathrm{t}},
\end{aligned}
$$

where $\boldsymbol{e}_{\mathrm{n}}$ is the normal direction of the fracture plane and $\boldsymbol{e}_{\mathrm{t}}$ is the tangential direction of the fracture plane. Because the normal component of the current and the tangential

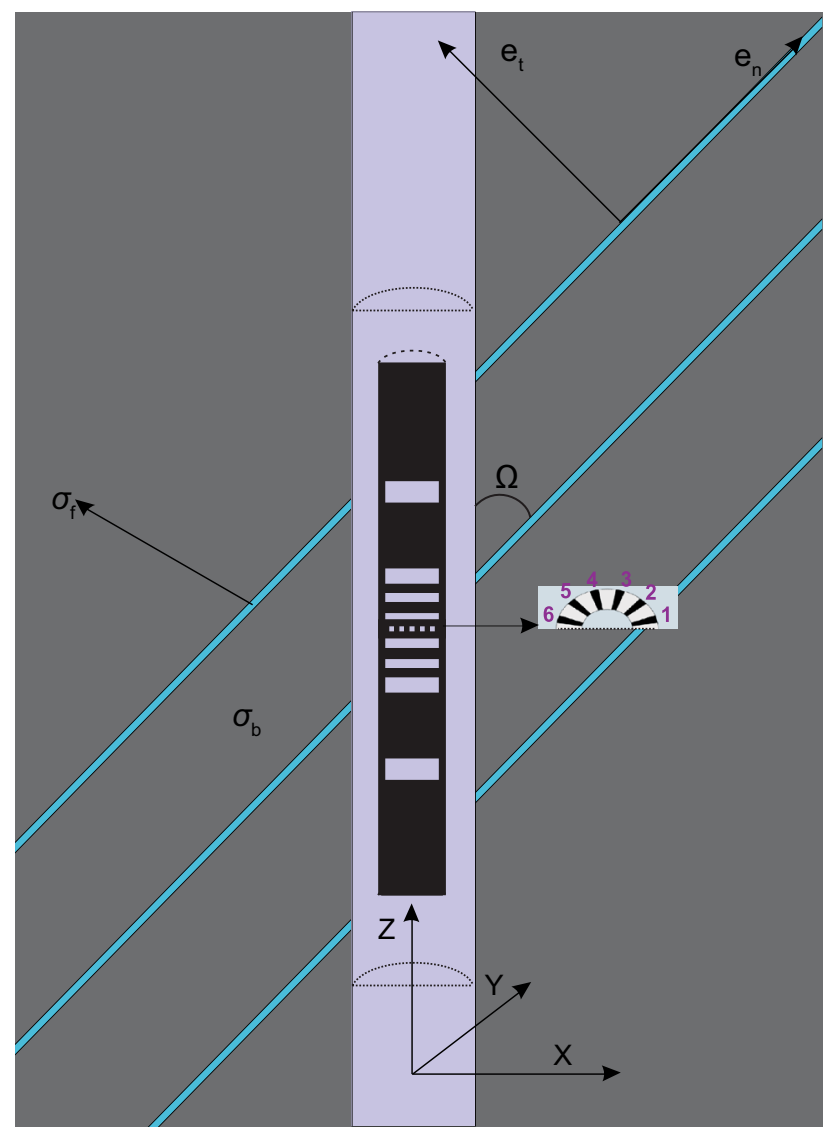

Fig. 2 Fractured reservoir model component of the potential in both sides of the fracture plane are continuous, the current density $\left(\boldsymbol{J}_{\mathrm{f}}\right)$ of fracture part can be decomposed into normal $\left(\boldsymbol{J}_{\mathrm{fn}}\right)$ and tangential $\left(\boldsymbol{J}_{\mathrm{ft}}\right)$ components which are given by

$\boldsymbol{J}_{\mathrm{fn}}=\sigma_{\mathrm{b}} \boldsymbol{e}_{\mathrm{n}}\left(-\nabla U \cdot \boldsymbol{e}_{\mathrm{n}}\right)=\sigma_{\mathrm{b}}\left(U_{x} \sin \Omega-U_{z} \cos \Omega\right) \boldsymbol{e}_{\mathrm{n}}$

$\boldsymbol{J}_{\mathrm{ft}}=\sigma_{\mathrm{f}}\left[-\nabla U-\left(U_{x} \sin \Omega-U_{z} \cos \Omega\right) \boldsymbol{e}_{\mathrm{n}}\right]$.

The formula of using the three-dimensional finite element method to simulate the logging response is given below:

$\Phi=\frac{1}{2} \iiint_{V} \boldsymbol{J} \cdot \boldsymbol{E} \mathrm{d} x \mathrm{~d} y \mathrm{~d} z-\sum_{E} U_{E} \boldsymbol{I}_{E}$,

where $\Phi$ is the energy functional, $V$ is the area of the threedimensional space minus the electrode system, $U_{E}$ is the potential of all electrodes, and $I_{E}$ is the supply current. According to Eqs. (5)-(9), the functional equations of HARL in fractured formations can be written as

$\Phi=\Phi_{\mathrm{b}}+\Phi_{\mathrm{f}}-\sum_{E} U_{E} I_{E}$

$\Phi_{\mathrm{f}}=\frac{1}{2} \iiint_{V_{\mathrm{f}}}\left[\left(\sigma_{\mathrm{b}}^{2} / \sigma_{\mathrm{f}}-\sigma_{\mathrm{f}}\right)\left(-U_{x} \sin \Omega+U_{z} \cos \Omega\right)^{2}\right.$

$$
\left.+\sigma_{\mathrm{f}}(\nabla U)^{2}\right] \mathrm{d} V_{\mathrm{f}}
$$

$\Phi_{\mathrm{b}}=\frac{1}{2} \iiint_{V_{\mathrm{b}}} \sigma_{\mathrm{b}}(\nabla U)^{2} \mathrm{~d} V_{\mathrm{b}}$,

where the integration function of $\Phi_{\mathrm{b}}$ is only in bedrock and the integration function of $\Phi_{\mathrm{f}}$ is only in fractures. Generally, $\phi_{\mathrm{f}}<<1$ and $\sigma_{\mathrm{f}}>>\sigma_{\mathrm{b}}$ are established for fracture porosity and fracture fluid conductivity in fractured reservoir, respectively. So Eq. (12) can be written as

$$
\begin{aligned}
\Phi= & \frac{1}{2} \iiint_{V}\left[\left(\sigma_{\mathrm{b}}+\sigma_{\mathrm{f}} \phi_{\mathrm{f}} \cos ^{2} \Omega\right)\left(U_{x}\right)^{2}+\left(\sigma_{\mathrm{b}}+\sigma_{\mathrm{f}} \phi_{\mathrm{f}}\right)\right]\left(U_{y}\right)^{2} \\
& +\left(\sigma_{\mathrm{b}}+\sigma_{\mathrm{f}} \phi_{\mathrm{f}} \sin ^{2} \Omega\right)\left(U_{z}\right)^{2} \\
& \left.+2 \sigma_{\mathrm{f}} \phi_{\mathrm{f}} \sin \Omega \cos \Omega U_{x} U_{z}\right] \mathrm{d} x \mathrm{~d} y \mathrm{~d} z-\sum_{E} U_{E} I_{E},
\end{aligned}
$$

where $\phi_{\mathrm{f}}$ is the fracture porosity. According to Eq. (13) and the specific boundary conditions, we can use an improved frontal solver to rapidly calculate the response of HARL (Zhang 1984).

\section{HARL response of fracture intersecting borehole}

\subsection{Calculation condition}

The borehole diameter is 8 inches. Mud resistivity is $1 \Omega \mathrm{m}$. Fracture fluid resistivity is $1 \Omega \mathrm{m}$. The numerical simulation 
model is axisymmetric. So we only observe six azimuthal resistivity curves, and the other six azimuthal resistivity curves are symmetrical about the symmetry plane. As shown in Fig. 2, the positive direction of the $x$-axis is set as the initial direction of the zero degree angle, and then the measured angle of the 1st-6th azimuthal resistivity successively increases. Because the response characteristics of deep and shallow azimuthal resistivities are similar, except in special circumstances, the article only shows deep azimuthal resistivity.

\subsection{Relationship of HARL response and fracture dipping angle}

The relationship of HARL response and different fracture dipping angles is shown in Fig. 3a, b, in which the
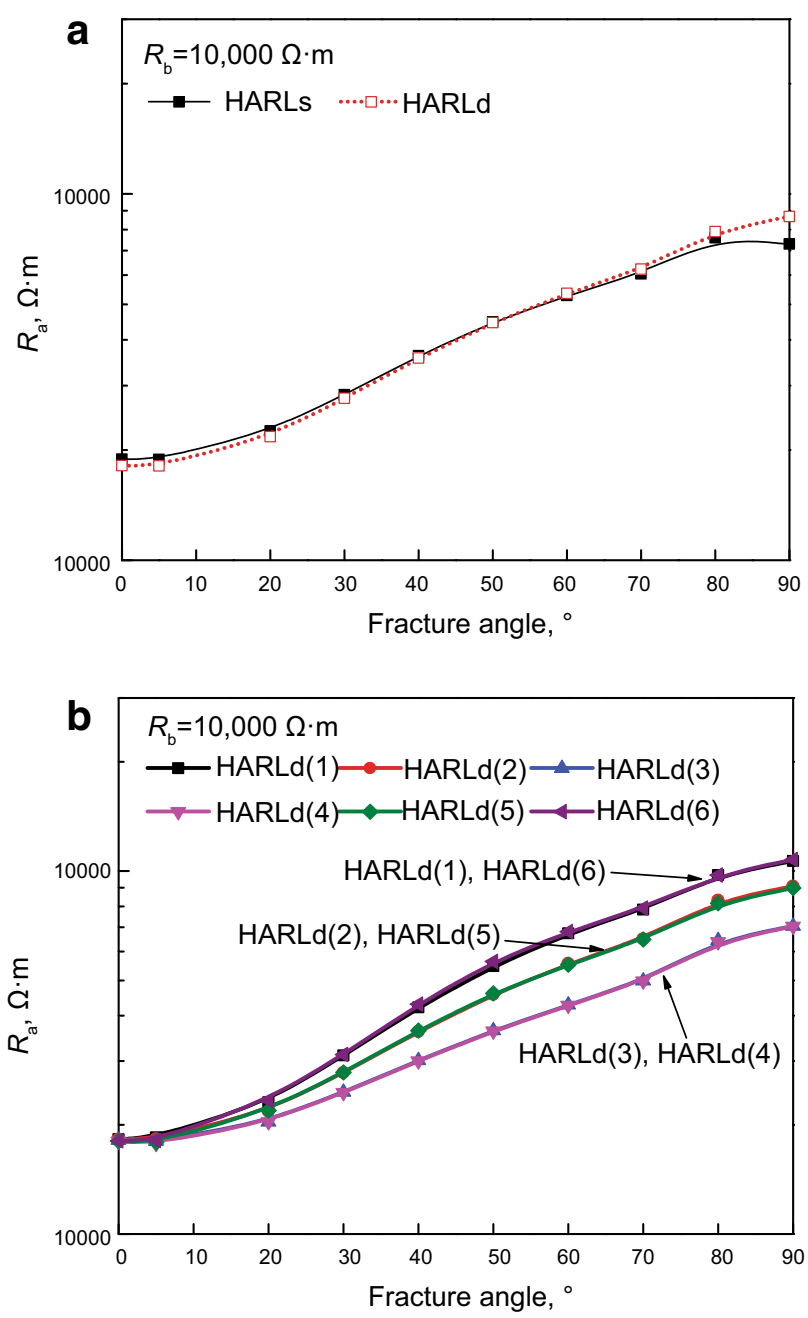

Fig. 3 Relationship of HARL response and different fracture dipping angles. a high-resolution dual laterolog curves, b deep azimuthal resistivity curves fracture aperture is $50 \mu \mathrm{m}$ and the bedrock resistivity is $10,000 \Omega \mathrm{m}$. Negative separations between the deep and shallow resistivity occur in low-angle fractures, while positive separations occur in high-angle fractures. As the fracture dipping angle increases, the apparent resistivity and the variation of azimuthal resistivity increase. The 3rd and 4th azimuthal resistivity become the lowest when the two azimuthal electrodes are close to the fracture strike direction. The 1 st and 6 th azimuthal resistivity become the highest when the two azimuthal electrodes are close to the fracture dipping direction. The results indicate that high-resolution dual laterolog curves can reflect the fracture dipping angle, and azimuthal resistivity curves contain information of the fracture dipping direction.

\subsection{Relationship of HARL response and fracture aperture}

The relationship of the apparent resistivity in high-resolution dual laterolog mode and different fracture aperture is shown in Fig. 4a. As the fracture aperture increases, the apparent resistivity decreases. The decrease rate is larger for horizontal fractures than for vertical fractures. So the apparent resistivity of low-angle fractures is obviously lower than that of high-angle ones with the same fracture aperture. When the fracture aperture is large, there are negative separations for low-angle fractures and positive separations for high-angle fractures. The corrected apparent conductivity means the part of the bedrock conductivity minus the logging apparent conductivity. As shown in Fig. $4 b$, the bedrock resistivity is $3000 \Omega \mathrm{m}$, and the dipping angles are respectively $0^{\circ}, 45^{\circ}$, and $90^{\circ}$. The corrected apparent conductivities of deep and shallow laterologs are almost linearly related to the fracture aperture. Apparent conductivities increase as the fracture aperture increases. The conductivity of the horizontal fracture is large; in terms of dual laterolog, the response is stronger for lowangle fractures than for high-angle fractures. The deep conductivity is larger than the shallow conductivity for low-angle fractures, while for high-angle fractures the results are opposite. The bigger the fracture aperture is, the larger the separation between the deep and shallow conductivities becomes.

The relationship of azimuthal resistivity curves and different fracture dipping angles is shown in Fig. 4c, d. The azimuthal resistivity curves of horizontal fractures are completely overlapped. The variation of azimuthal resistivity becomes larger as the fracture aperture and the 

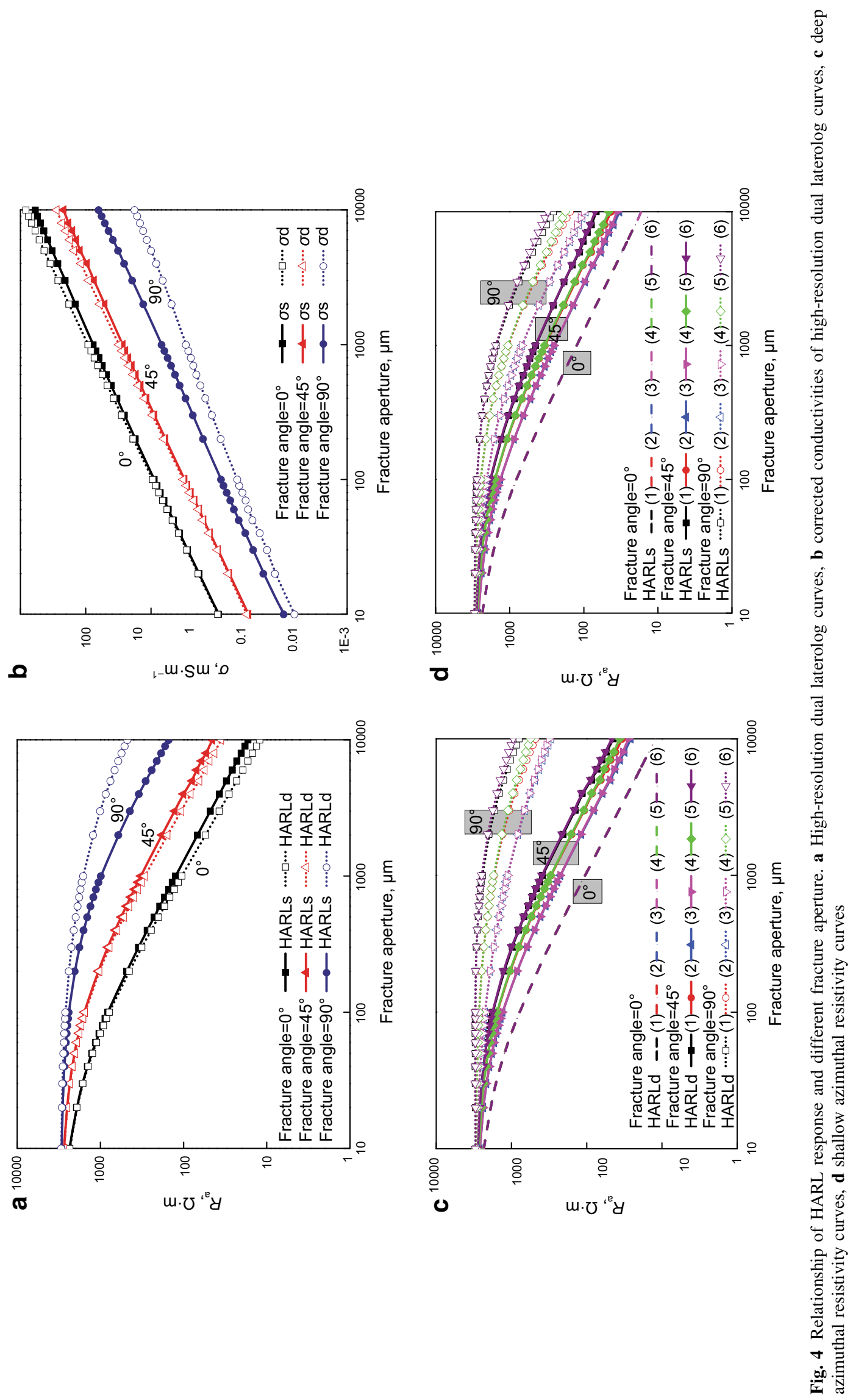
fracture dipping angle increase, which reaches maximum as the fracture dipping angle reaches $90^{\circ}$.

\subsection{Azimuthal resistivity imaging of fractures}

The azimuthal resistivity imaging of fractures with different dipping angles is shown in Fig. 5a. From top to bottom, fracture dipping angles are respectively $0^{\circ}, 45^{\circ}$, and $75^{\circ}$; the bedrock resistivity is $3000 \Omega \mathrm{m}$; and the fracture aperture is $200 \mu \mathrm{m}$. As the fracture dipping angle increases, the bending degree of sine curves becomes bigger and the apparent aperture of fractures becomes larger. When the aperture of fractures is small, the identification of fractures, especially high-angle fractures, becomes challenging, as shown in Fig. $5 \mathrm{~b}$, in which the aperture of fractures reduces to $5 \mu \mathrm{m}$ with the same other conditions as in Fig. 5a.

When using the azimuthal resistivity to identify fractures, low-angle fractures can be identified by the decreased amplitude of apparent resistivity, and high-angle fractures can be identified by the variation of azimuthal resistivity. Assuming that the resistivity ratio of bedrock and fracture fluid is $3000: 1$, for low-angle fractures, the smallest detectable aperture can be less than $10 \mu \mathrm{m}$ when we set $10 \%$ (the dotted line in Fig. 6a) as an identification threshold in terms of the ratio of the resistivity of the fracture and the bedrock (the formula in Fig. 6a). For high-angle fractures, the smallest detectable aperture can be larger than $10 \mu \mathrm{m}$ in terms of the ratio of the maximum variation of the azimuthal resistivity and the laterolog resistivity (the formula in Fig. 6b). For medium-angle fractures, the smallest detectable aperture is also larger than $10 \mu \mathrm{m}$ considering both resistivity amplitude difference and azimuthal variation, as the formulas and the dotted lines shown in Fig. 6c, d. When the resistivity ratio increases to $10,000: 1$, according to all the solid lines shown in Fig. 6, the detectable aperture becomes smaller. When the fracture aperture is large enough, the maximum variation no longer significantly increases and even decreases for medium-angle and high-angle fractures, as shown in Fig. $6 \mathrm{~b}$, d. The spatial resolution of the FMI-HD tool is 0.2 in $(5.08 \mathrm{~mm})$, representing the button size of each electrode. But the highresolution electrodes are sensitive enough to identify fluidfilled fractures less than $10 \mu \mathrm{m}$ in width.

\section{HARL response of crossing fractures}

A fracture group usually exists in the form of crossing fractures. This article only discusses the situation of two crossing fractures, as shown in Fig. 7 , in which the bedrock resistivity is $10,000 \Omega \mathrm{m}$ and the fracture aperture is $50 \mu \mathrm{m}$. Assuming that one arbitrary dipping angle fracture respectively crosses with one horizontal fracture and one vertical fracture, by comparing their responses (solid lines) with the response of parallel fractures (dotted lines), the apparent resistivity of crossing fractures increases more slowly, as shown in

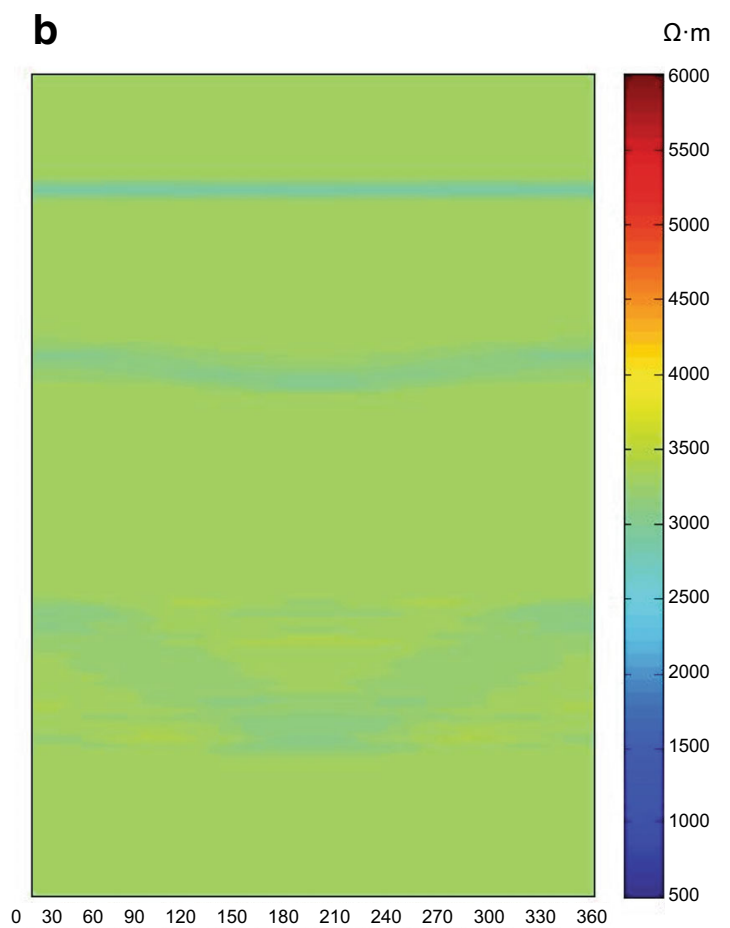

Fig. 5 Deep azimuthal resistivity imaging of fractures with different dipping angles. a Fracture aperture $200 \mu \mathrm{m}, \mathbf{b}$ fracture aperture $5 \mu \mathrm{m}$ 

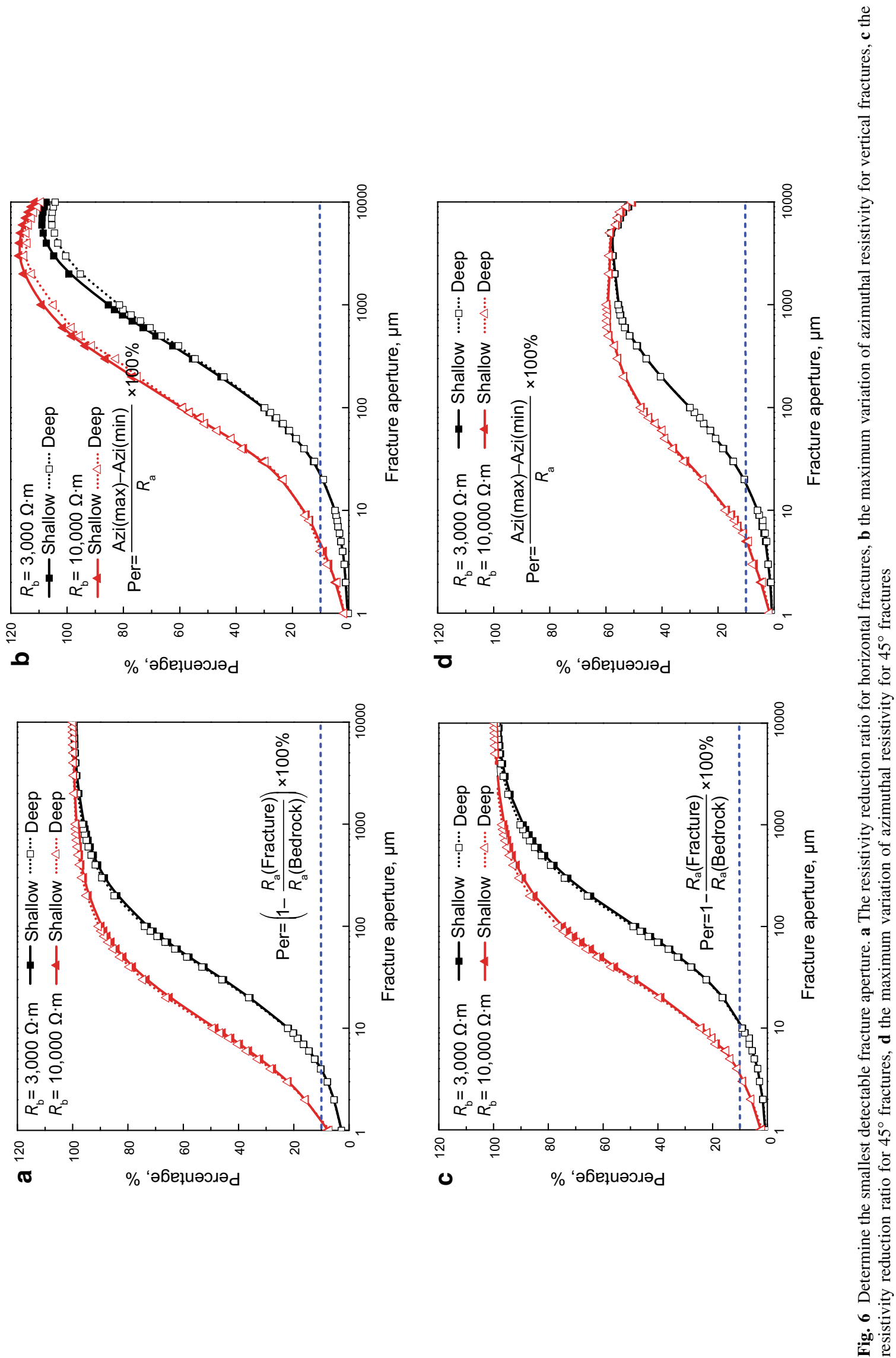

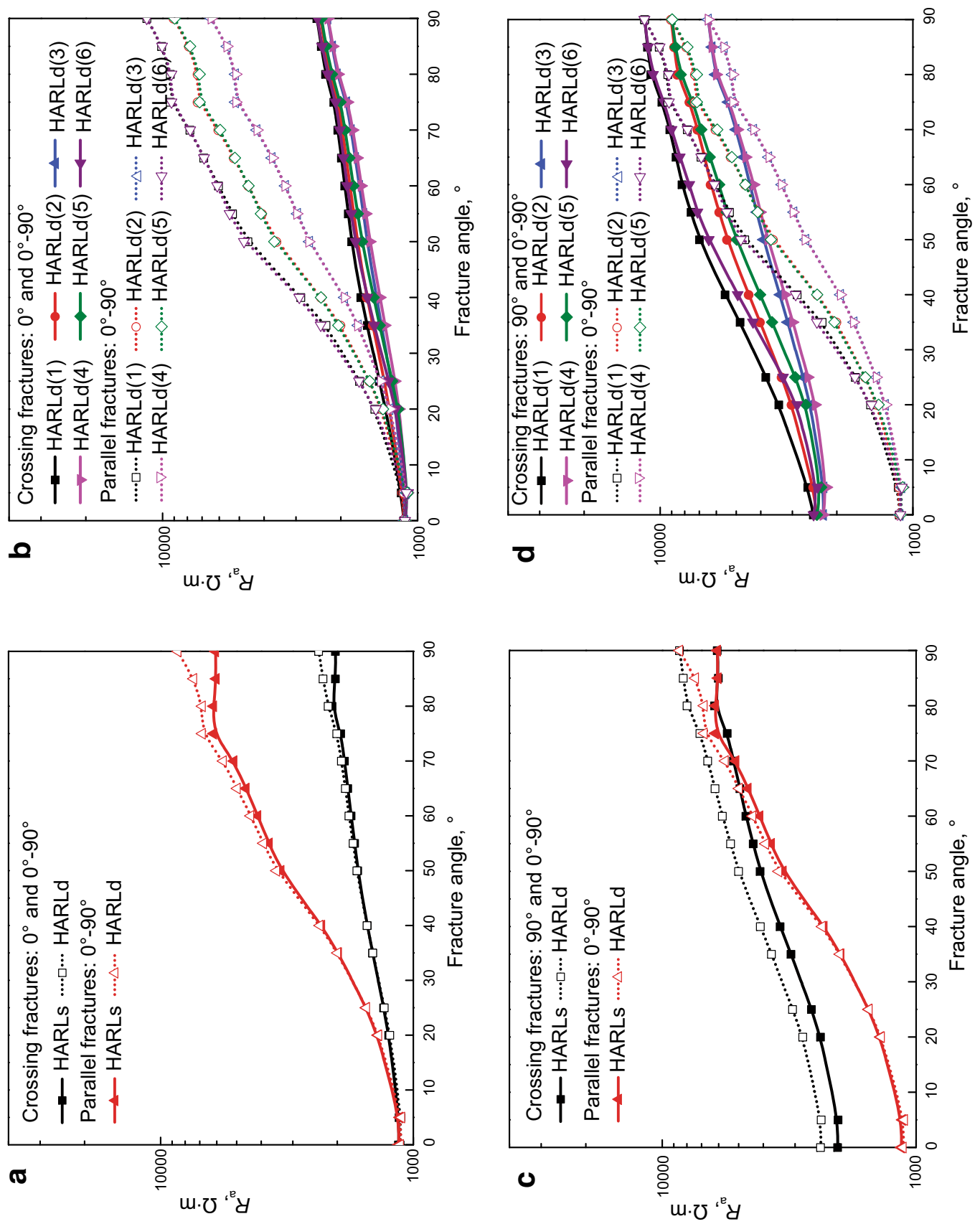
a

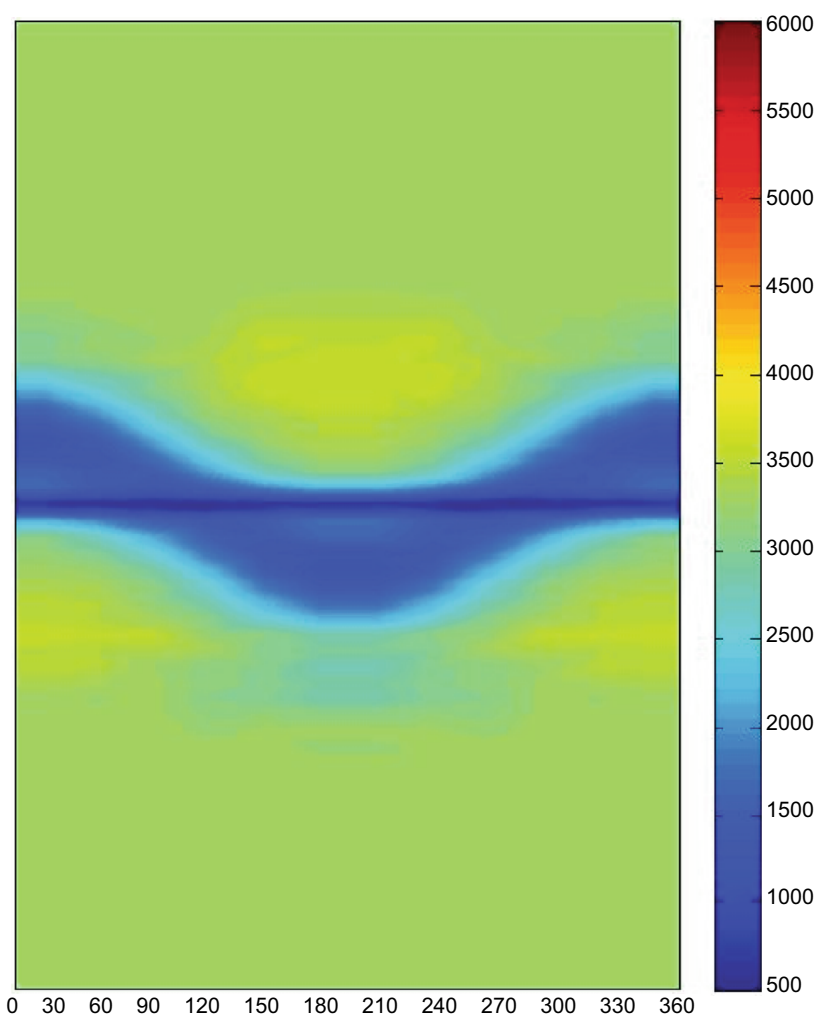

b

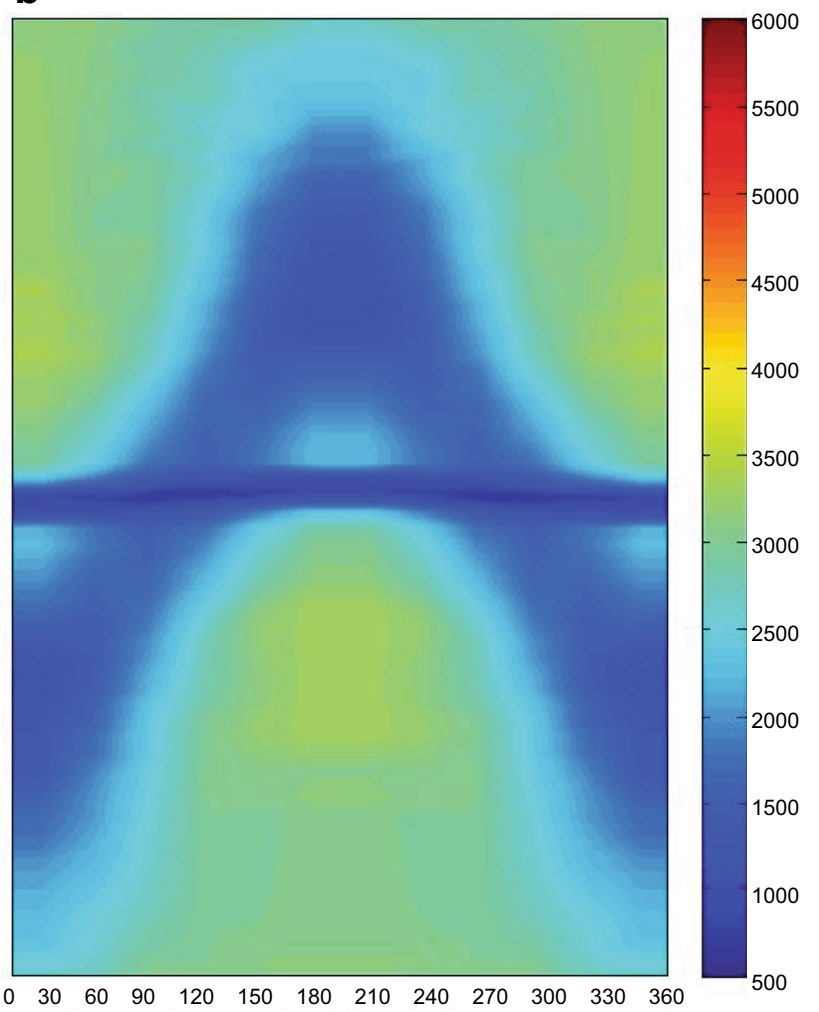

Fig. 8 Azimuthal resistivity imaging of crossing fractures. a Deep azimuthal resistivity imaging of $0^{\circ}$ and $75^{\circ}$ crossing fractures, b shallow azimuthal resistivity imaging of $120^{\circ}$ and $170^{\circ}$ crossing fractures

Fig. 7 a, c. In comparison, the variation of azimuthal resistivity of crossing fractures that include horizontal fracture is weaker and those that include vertical fracture gradually increase as the dipping angle increases, as shown in Fig. $7 b$, d.

Figure 8a shows a deep azimuthal resistivity imaging of crossing fractures with one horizontal and one $75^{\circ}$ highangle fracture, and Fig. 8b shows a shallow azimuthal resistivity imaging of crossing fractures with one $120^{\circ}$ and one $170^{\circ}$ fracture, in which the bedrock resistivity is $3000 \Omega \mathrm{m}$, and the fracture aperture is $200 \mu \mathrm{m}$. The results show that, in crossing fractures, the apparent resistivity is mainly affected by the low-angle fracture, while the variation of azimuthal resistivity is mainly affected by the high-angle fracture. The combination type of crossing fractures can be clearly displayed.

\section{HARL response of high-angle fracture beside the borehole}

Fracture and structure prediction beside the borehole is very important, but it is challenging for conventional logging. The HARL response is utilized to study vertical fractures beside the borehole, and the apparent resistivities are shown in Fig. 9a, c, and e, in which the fracture apertures are 50, 200, and $1000 \mu \mathrm{m}$, and the bedrock resistivities are 10,000 and $3000 \Omega \mathrm{m}$, respectively. When the vertical fracture intersects the borehole, which means that the distance from the fracture to the center of borehole is less than $0.1 \mathrm{~m}$, the apparent resistivity is significantly small and a positive separation occurs between the deep and shallow resistivities. As the vertical fracture is gradually far away from the borehole, the positive separation gradually disappears. For the vertical fracture far from the borehole, if the fracture aperture and the resistivity ratio are large enough, negative separation occurs. This is because the impact of deep laterolog is greater than that of the shallow one, resulting in faster decrease of the deep lateral resistivity.

The azimuthal resistivity response is shown in Fig. 9b, $\mathrm{d}$, and $\mathrm{f}$. When the vertical fracture intersects the borehole, the azimuthal electrode closest to the fracture plane has the minimal resistivity, and the variation of azimuthal resistivity is obvious. When the vertical fracture is near the sidewall, the variation is the largest. As the vertical fracture is gradually far away from the borehole, the variation gradually weakens. As the distance between the 1st azimuthal electrode and vertical fracture is the nearest, it has the smallest resistivity, and the 6th 

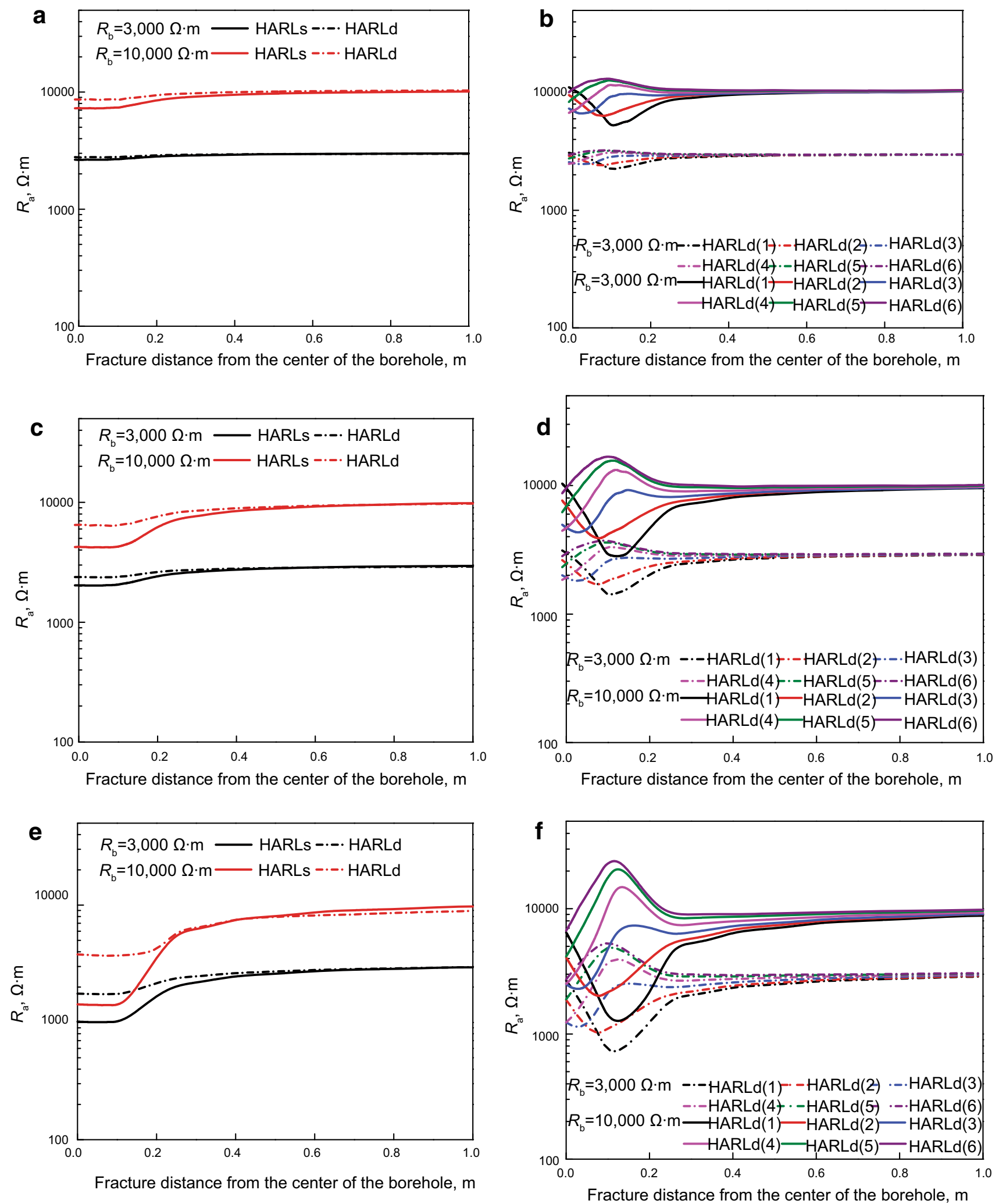

Fig. 9 HARL response of vertical fracture with different distances from the center of borehole. a High-resolution dual laterolog curves of a $50 \mu \mathrm{m}$ fracture, b deep azimuthal resistivity curves of a $50 \mu \mathrm{m}$ fracture, $\mathbf{c}$ high-resolution dual laterolog curves of a $200 \mu \mathrm{m}$ fracture, $\mathbf{d}$ deep azimuthal resistivity curves of a $200 \mu \mathrm{m}$ fracture, e high-resolution dual laterolog curves of a $1000 \mu \mathrm{m}$ fracture, f deep azimuthal resistivity curves of a $1000 \mu \mathrm{m}$ fracture 


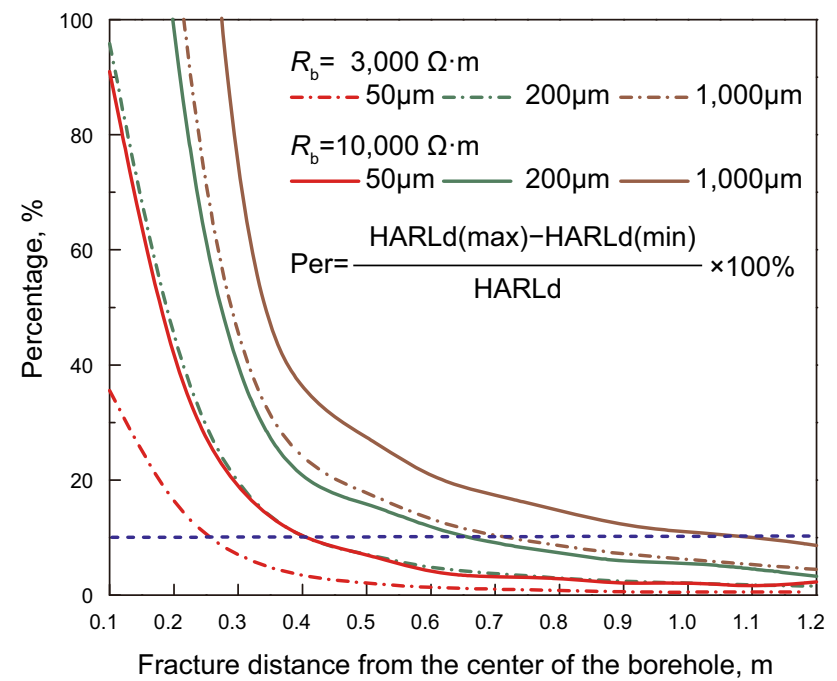

Fig. 10 Determination of the farthest detectable distance of a vertical fracture beside the borehole

electrode has the largest resistivity because of its farthest distance.

For vertical fractures beside the borehole, the farthest detectable distance is determined as the ratio of the maximum variation of deep azimuthal resistivity and the deep lateral resistivity that reaches $10 \%$. As shown in Fig. 10, when the bedrock resistivity is $3000 \Omega \mathrm{m}$ and the fracture apertures are 50, 200, and $1000 \mu \mathrm{m}$, the farthest detectable distances are $0.25,0.40$, and $0.7 \mathrm{~m}$, respectively. When the bedrock resistivity is $10,000 \Omega \mathrm{m}$, and the fracture apertures are 50, 200, and $1000 \mu \mathrm{m}$, the farthest detectable distances are $0.40,0.67$, and $1.1 \mathrm{~m}$, respectively.

\section{Conclusions}

A good correlation exists between HARL and fracture aperture. A relatively low apparent resistivity and negative separations between deep and shallow resistivities present for low-angle fractures, while a relatively high azimuthal resistivity and positive separations exist for high-angle fractures.

Azimuthal resistivity imaging can be used to identify the presence of the fractures and their combination type. As the fracture dipping angle, aperture, or the resistivity ratio increases, the variation of azimuthal resistivity increases.

According to the positive or negative separations between the deep and shallow resistivities and the variation of azimuthal resistivity, high-angle fractures surrounding the borehole can be determined when the fracture aperture is relatively large and the resistivity ratio of bedrock and fracture medium is high.
Acknowledgments This research has been co-funded by the National Natural Science Foundation of China (41174099, 41474100), the Fundamental Research Funds for the Central Universities (14CX06077A), and National Major Science \& Technology Projects of China (2011ZX05003, 2011ZX05009, 2011ZX05020, 2011ZX 05035).

Open Access This article is distributed under the terms of the Creative Commons Attribution License which permits any use, distribution, and reproduction in any medium, provided the original author(s) and the source are credited.

\section{References}

Bourbiaux B. Fractured reservoir simulation: a challenging and rewarding issue. Oil \& Gas Sci Technol-Rev IFP. 2010;65(2): 227-38.

Davies DH, Faivre O, Gounot MT, et al. Azimuthal resistivity imaging: a new generation laterolog. SPE Form Eval. 1994;9(03):165-74 SPE 24676.

Deng SG, Li ZQ. Simulation of array laterolog response of fracture in fractured reservoir. Earth Sci: J China Univ Geosci. 2009;34(5): 841-7 (in Chinese).

Deng SG, Mo XX, Lu CL, et al. Numerical simulation of the dual laterolog response to fractures and caves in fractured-cavernous formation. Pet Explor Dev. 2012;39(6):751-7 (in Chinese).

Deng SG, Wang Y, Hu YY, et al. Integrated petrophysical log characterization for tight carbonate reservoir effectiveness: a case study from the Longgang area, Sichuan Basin, China. Pet Sci. 2013;10(3):336-46.

Dershowitz WS, Cottrell MG, Lim DH, et al. A discrete fracture network approach for evaluation of hydraulic fracture stimulation of naturally fractured reservoirs. In: 44th U.S. rock mechanics symposium and 5th U.S.-Canada rock mechanics symposium, June 27-30, Salt Lake City, Utah (ARMA 10475); 2010.

Faivre O. Fracture evaluation from quantitative azimuthal resistivity. In: Annual technical conference and exhibition. SPE annual technical conference and exhibition, October 3-6, Houston, Texas (SPE 26434); 1993.

Ja'fari A, Kadkhodaie-Ilkhchi A, Sharghi Y, et al. Fracture density estimation from petrophysical log data using the adaptive neurofuzzy inference system. J Geophys Eng. 2012;9(1):105.

Jiang LZ, Gu JY, Guo BC. Characteristics and mechanism of low permeability clastic reservoir in Chinese petroliferous basin. Acta Sedimentol Sin. 2004;22(1):13-8 (in Chinese).

Karim SM, Murugesu TS and Li B. The utilisation of high-definition borehole images to determine fracture properties and their relative age for fractured basement reservoirs. In: International petroleum technology conference, March 26-28, Beijing, China (IPTC 17144); 2013.

Kuchuk F, Biryukov D. Pressure-transient behavior of continuously and discretely fractured reservoirs. SPE Reserv Eval Eng. 2014; 17(1):82-97 (SPE 158096).

Le F, Zhou ZQ, Maurer HM, et al. 3-D study of resistivity tool responses in formations with vertical fractures or horizontal transverse isotropy. In: SPWLA 52nd annual logging symposium, May 14-18, Colorado Springs, Colorado; 2011.

Li SJ, Xiao CW, Wang HM, et al. Mathematical model of dual laterolog response to fracture and quantitative interpretation of fracture porosity. Acta Geophysi Sin. 1996;39(6):845-52 (in Chinese).

Moinfar A, Varavei A, Sepehrnoori K, et al. Development of a novel and computationally-efficient discrete-fracture model to study IOR processes in naturally fractured reservoirs. In: SPE 
improved oil recovery symposium, April 14-18, Tulsa, Oklahoma, USA (SPE 154246); 2012.

Nie RS, Meng YF, Jia YL, et al. Dual porosity and dual permeability modeling of horizontal well in naturally fractured reservoir. Transp Porous Media. 2012;92(1):213-35 (SPE 11242).

Noroozi MM, Moradi B and Bashiri G. Effects of fracture properties on numerical simulation of a naturally fractured reservoir. In: Trinidad and tobago energy resources conference, June 27-30, Port of Spain, Trinidad (SPE 132838); 2010.

Olsen TN, Germinario MP, Reinmiller R, et al. Horizontal lateral image analysis applied to fracture stage optimization in Eastern Barnett Shale, Tarrant and Dallas Counties, Texas. In: SPE/AAPG/SEG unconventional resources technology conference, August 25-27, Denver, Colorado, USA (SPE 1922149); 2014.

Reynolds MM, Bachman RC and Peters WE. A comparison of the effectiveness of various fracture fluid systems used in multistage fractured horizontal wells: Montney Formation unconventional gas. In: SPE hydraulic fracturing technology conference, February, 4-6, The Woodlands, Texas, USA (SPE 168632); 2014.

Sausse J, Dezayes C, Dorbath L, et al. 3D model of fracture zones at Soultzsous-Forêts based on geological data, image logs, induced microseismicity and vertical seismic profiles. CR Geosci. 2010;342(7): $531-45$.

Shen J, Su B, Guo N. Anisotropic characteristics of electrical responses of fractured reservoir with multiple sets of fractures. Pet Sci. 2009;6(2):127-38.

Sibbit AM and Faivre O. The dual laterolog response in fractured rocks. In: SPWLA 26th annual logging symposium, June 17-20, Dallas, Texas; 1985.

Smits JW, Benimeli D, Dubourg I, et al. High resolution from a new laterolog with azimuthal imaging. In: Society of petroleum engineers annual technical conference. 1995, p. 563-76 (SPE 30584).
Sun SZ, Zhou XY, Yang HJ, et al. Fractured reservoir modeling by discrete fracture network and seismic modeling in the Tarim Basin, China. Pet Sci. 2011;8(4):433-45.

Weng X, Kresse O, Cohen CE, et al. Modeling of hydraulic-fracturenetwork propagation in a naturally fractured formation. Soc Pet Eng. 2011;26(04):368-80 (SPE 140253).

Yang HJ, Sun SD, Cai LL, et al. A new method of formation evaluation for fractured and caved carbonate reservoirs: a case study from the Lundong area, Tarim Basin, China. Pet Sci. 2011; 8(4):446-54.

Yang W and Tao G. Forward and inversion of azimuthal lateral resistivity logs. In: SEG annual meeting, 31 October-5 November, Houston, Texas (SEG 1999-0120); 1999.

Yao J, Sun H, Fan DY, et al. Numerical simulation of gas transport mechanisms in tight shale gas reservoirs. Pet Sci. 2013;10(4): 528-37.

Yun Y, Hou HJ, Hui $\mathrm{H}$, et al. Understanding reservoir properties through high-definition microresistivity images in horizontal shale oil wells drilled with OBM in China. In: SPE middle east oil and gas show and conference, March 10-13, Manama, Bahrain (SPE 164360); 2013.

Zeng LB, Li YG, Wang ZG, et al. Type and sequence of fractures in the second member of Xujiahe Formation at the south of western Sichuan Depression. Earth Sci: J China Univ Geosci. 2007;32(2): 194-200 (in Chinese).

Zhang GJ. Electrolog (I). Beijing: Petroleum Industry Press; 1984. p. 1-38 (in Chinese).

Zhang Z, Tong HM, Bao ZD. Development characteristics and quantitative prediction of reservoir fractures in the Chaoyanggou oil field. Min Sci Technol (China). 2009;19(3):373-9.

Zhao XM, Liu L, Hu JL, et al. The tectonic fracture modeling of an ultra-low permeability sandstone reservoir based on an outcrop analogy: a case study in the Wangyao Oilfield of Ordos Basin, China. Pet Sci. 2014;11(3):363-75. 